\title{
Method to assess lateral handling qualities of aircraft with wingtip morphing
}

\author{
Gaétan Dussart*, Sezsy Yusuf ${ }^{\dagger}$, Vilius Portapas ${ }^{\ddagger}$, \\ Guillermo Lopez ${ }^{\S}$ and Mudassir Lone \\ Cranfield University, Dynamic Simulation and Control Group \\ Cranfield, Bedfordshire, United Kingdom, MK43 OAL
}

\begin{abstract}
The impact of in-flight folding wingtip on roll characteristics of aircraft has been studied in the past. In this study, a handling qualities assessment carried out to de-risk further development of such a device. A specialised flight simulation campaign is prepared to evaluate the roll dynamics in different morphing configurations. Various manoeuvres, including the Offset Landing Manoeuvre and herein presented Slalom and Alignment Tracking task are used. Cooper Harper Rating scales and flight data analysis are used to collect pilot opinion and validate pilot-in-the-loop simulation results. This example is used to demonstrate the use of the slalom and Alignment Tracking manoeuvre for lateral dynamic assessment.
\end{abstract}

\section{Introduction}

A means to improve aircraft performance is to increase aircraft wingspan and raise aerodynamic efficiency. ${ }^{1,2}$ Then to comply with airport infrastructure limits, ground folding wingtips can be implemented as a solution, as seen on the NASA SUGAR concept (Fig.1a) or the Boeing 777X aircraft (Fig.1b). Moreover, in-flight folding can be considered to further justify the mechanism weight penalty and consolidate concept's viability. Two key applications were initially identified: gust loads alleviation capability demonstrated in past work, ${ }^{1}$ and flight dynamic and performance modifications, carried out through the preliminary identification of effect on key aerodynamic derivatives. ${ }^{3}$ This particular research demonstrated shifts of noticeable amplitude and trends in key lateral aerodynamic derivatives of a large generic aircraft. Despite small changes proportional to the baseline value, pilot-in-the-loop simulations are still necessary for assessing the suitability of this mechanism and its impact on handling qualities. Handling qualities can be understood as the ease with which a task can be performed by a pilot, within a given scope or environment. ${ }^{4}$ The assessment of handling qualities is a necessity to understand the manner in which the aircraft responds to a pilot or disturbance input. Aviation safety and regulations ${ }^{5,6}$ provide criteria and specifications through which aircraft are assessed. With the development of flight control augmentation systems, assessment of handling qualities has become more complex and distant from basic evaluation of aerodynamic stability and control. Control augmentation effects in fly-by-wire control systems now play a far greater role in enhancing handling qualities but rely on advanced control methods that in turn need accurate models, or in this case models that include corrections due to in-flight wingtip folding. These corrections must be developed through extensive flight testing and simulation; the latter relying heavily on flight simulators. An accurate flight simulator with appropriate aircraft models can help reduce time and cost of a flight test campaign. Characteristics of a particular machine and key development and design information can be derived from valuable measurements. Nonetheless, they should be considered as complementary and not supersede real-flight or wind tunnel testing in the development process. ${ }^{7}$

*PhD candidate, Dynamic Simulation and Control Group, g.x.dussart@cranfield.ac.uk

$\dagger \mathrm{PhD}$ candidate, Dynamic Simulation and Control Group, sezsy.yusuf@cranfield.ac.uk

$\ddagger$ PhD candidate, Dynamic Simulation and Control Group, v.portapas@cranfield.ac.uk

$\S$ PhD candidate, Dynamic Simulation and Control Group, g.lopez@cranfield.ac.uk

『Lecturer, Dynamic Simulation and Control Group, m.m.lone@cranfield.ac.uk 
This work aims at providing pilot-in-the-loop insight on the use of in-flight folding wingtips on a large generic aircraft, as illustrated in the overall research diagram in Fig.2. This would help prove the suitability of such a device and identify possible handling qualities deficiencies.

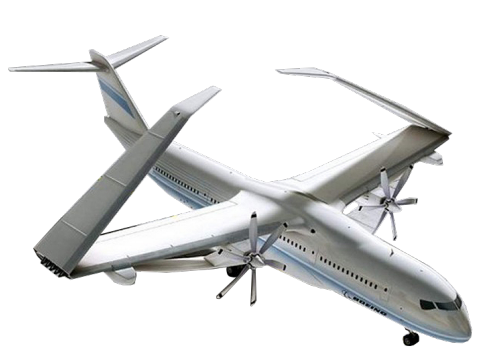

(a) Boeing SUGAR Concept ${ }^{8}$

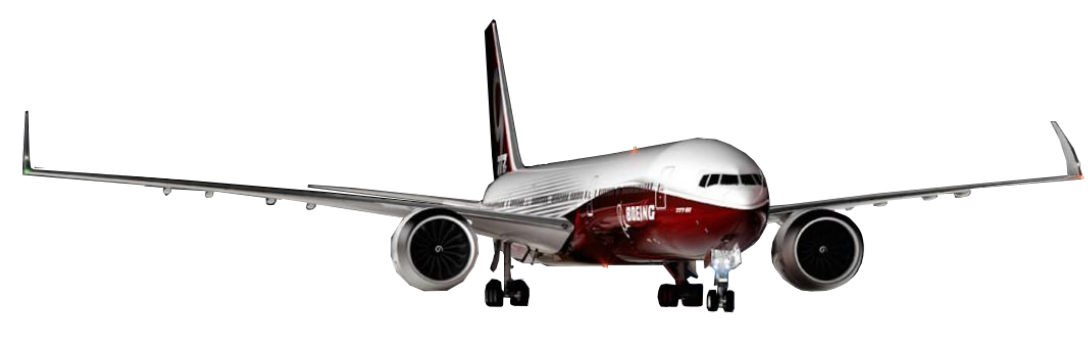

(b) Boeing $777 \mathrm{X}^{9}$

Figure 1: Ground dihedral morphing aircraft examples

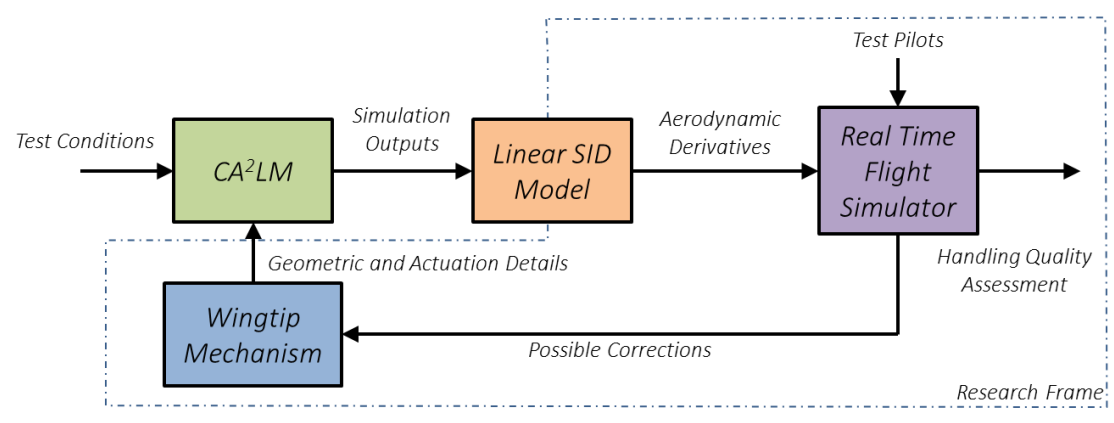

Figure 2: Research Frame

The paper aims at both proving the validity of the selected manoeuvres for roll dynamic assessment and de-risking the folding wingtip concept. Whilst this paper focuses on pilot-in-the-loop simulations, a brief description of the folding mechanism and aircraft is provided in Section II. The frameworks and methodologies used to obtain the relevant aerodynamic derivatives that characterise the roll dynamics are briefly introduced in Section III. The real time flight simulation environment considered for this investigation is then presented in Section IV before introducing the flight test campaign, the list of flight simulations and data processing methods considered for handling qualities assessment. A novel multi-directional manoeuvre, along with more conventional tasks such as the Offset Landing Manoeuvre ${ }^{10}$ are used to assess lateral handling qualities with morphing wingtip deflection.

\section{Dihedral wingtip morphing}

A dihedral morphing mechanism designed primarily for gust loads alleviation ${ }^{3}$ was implemented on a large generic aircraft as illustrated in Fig.3. This device is illustrated in Fig.4. The wingtip hinge is fixed to be perpendicular to the wing leading edge $\left(\Lambda^{\text {hinge }}=30^{\circ}\right)$ so as to decrease local effective wing angle of attack with upward morphing. ${ }^{1,3}$ The wingtip device is intended to be deflected during high loads encounters throughout the aircraft flight envelope. Hence, particular interest was given to morphing during low dynamic pressure conditions, i.e. take-off, ascent, descent and landing flight conditions where handling qualities are more critical. A set of 10 flight conditions, illustrated in Fig.5, were selected and two wingtip sizes, corresponding to $10 \%$ and $20 \%$ of wing semispan were studied to capture variations in lateral dynamics with wingtip length. To capture both normal operations and failure scenarios, controlled positions ranging 
from $\Gamma^{w t}=0^{\circ}$ to $\Gamma^{w t}=30^{\circ}$, as well as a loose wingtip positions were simulated. Additionally, the aircraft was flown both in the rigid flight and flexible shape at each flight conditions. Therefore, a sufficiently large test matrix was used to capture the effect of folding wingtips on lateral dynamics of the aircraft with mechanism characteristics and flight conditions.

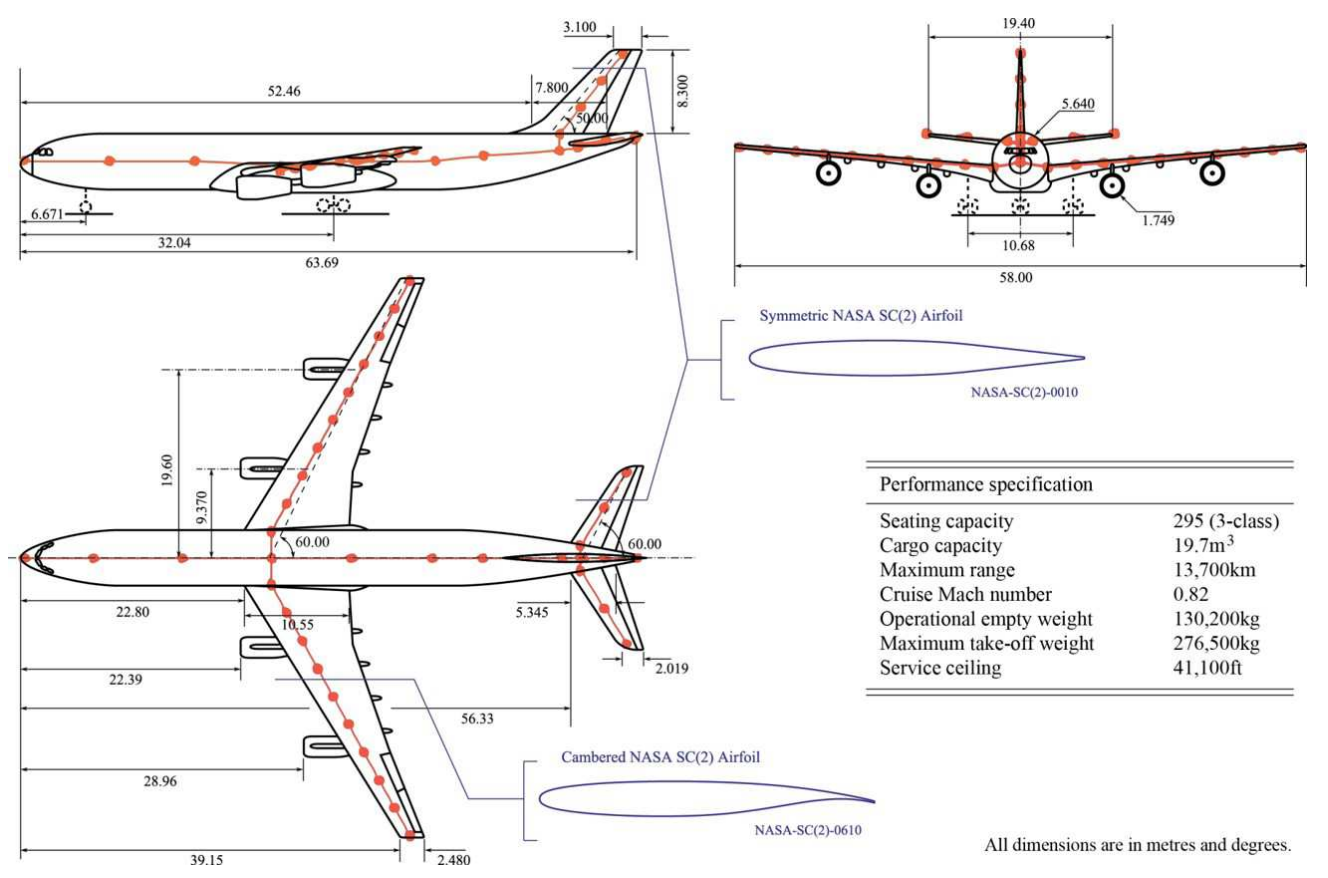

Figure 3: Cranfield University AX-1 long range aircraft details.

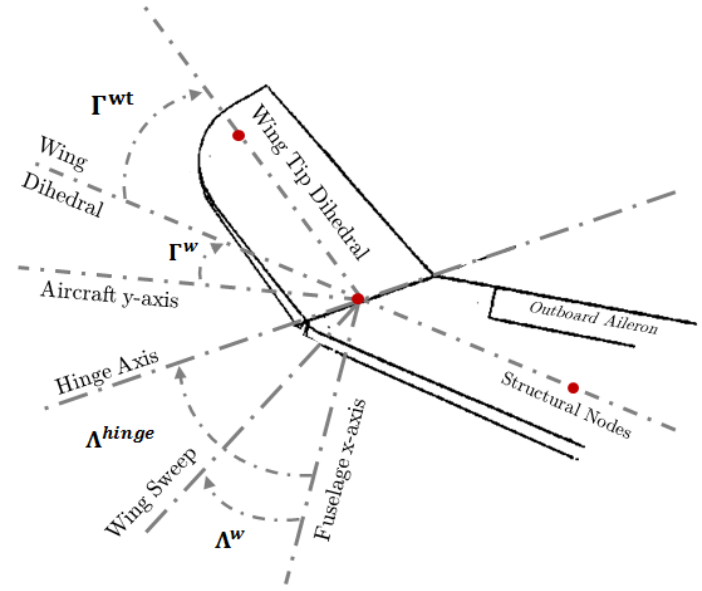

Figure 4: Illustration of the folding wingtip mechanism

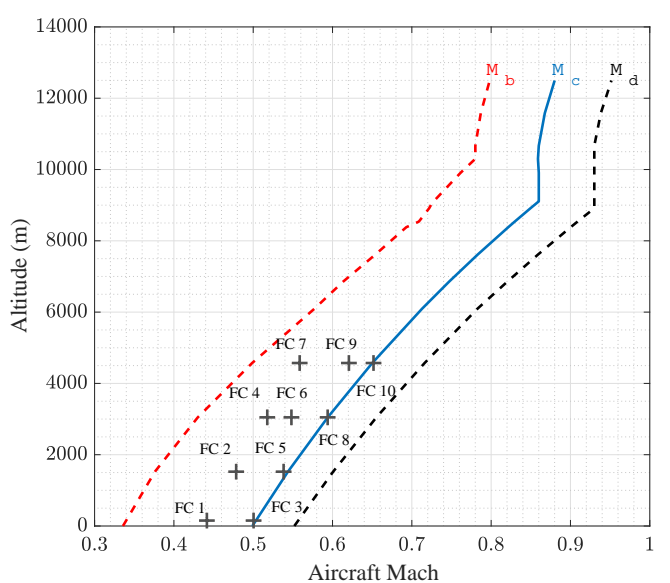

Figure 5: Selected flight conditions

\section{Capturing flight dynamic morphing effects}

\section{A. Simulation of roll dynamics}

To simulate aircraft response to an aileron input, and therefore capture the roll response, the folding wingtip mechanism was modelled within a time-domain 6 degrees of freedom (DoF) aeroservoelastic framework developed at Cranfield University. ${ }^{11-13}$ The framework relies on coupled unsteady aerodynamics and linear structural model, to compute the flexible aircraft dynamics for analysis. This near real-time framework was used to simulate small amplitude roll manoeuvres at the flight conditions defined in Fig.5, highlighting roll 
dynamics of the aircraft throughout a sufficiently large part of the flight envelope. As a physics based model, where aerodynamic loads and structural deformations are calculated at each time step, the framework does not rely on aerodynamic derivatives; metrics widely used within the flight dynamics community.

\section{B. Aerodynamic derivative definition}

As a 6 DoF system, the aircraft modes can be decoupled into longitudinal, lateral and directional axes. Aircraft flight dynamics can be predicted using linearised equations of motions, for small perturbations around an equilibrium or trim point. For example, the coupled lateral-directional dynamics can be modelled as follows:

$$
\left[\begin{array}{cccc}
m & 0 & 0 & 0 \\
0 & I_{x x} & -I_{x z} & 0 \\
0 & -I_{x z} & I_{z z} & 0 \\
0 & 0 & 0 & 1
\end{array}\right]\left[\begin{array}{c}
\dot{v} \\
\dot{p} \\
\dot{r} \\
\dot{\phi}
\end{array}\right]=\left[\begin{array}{cccc}
Y_{v} & Y_{p} & Y_{r} & Y_{\phi} \\
L_{v} & L_{p} & L_{r} & L_{\phi} \\
N_{v} & N_{p} & N_{r} & N_{\phi} \\
0 & 1 & 0 & 0
\end{array}\right]\left[\begin{array}{c}
v \\
p \\
r \\
\phi
\end{array}\right]+\left[\begin{array}{cc}
Y_{\xi} & Y_{\zeta} \\
L_{\xi} & L_{\zeta} \\
N_{\xi} & N_{\zeta} \\
0 & 0
\end{array}\right]\left[\begin{array}{l}
\xi \\
\zeta
\end{array}\right]
$$

where $m$ and $I$ represent aircraft mass and inertia respectively. $v$ is the lateral velocity, $p$ and $r$ are the roll and yaw rates and $\phi$ is the bank angle. Furthermore, the controls are represented by $\xi$ and $\zeta$ which correspond to aileron and rudder deflections respectively. The parameters $L_{\square}, N_{\square}$ and $Y_{\square}$ are aerodynamic derivatives. These can be described as a measure of how particular parameters such as flight conditions, aircraft attitude, rotation rates or forces and moments, influence the aircraft response at a given flight condition. A more extensive description of the aerodynamic derivatives can be found in Cook. ${ }^{14}$ In this case $L$ quantifies rolling moment whilst $N$ quantifies yawing moment. $\beta$ is the sideslip angle, which is defined as $\frac{v}{V}$, in which $V$ represents airspeed. Therefore, $v$ can be replaced by $\beta$ in Eqn.1 and the linear mathematical expression for the rolling characteristics can be written as :

$$
\dot{p}-\frac{I_{x z}}{I_{x x}} \dot{r}=L_{p} \Delta \frac{p b}{2 V}+L_{\xi} \Delta \xi+L_{r} \Delta \frac{r b}{2 V}+L_{\beta} \Delta \beta+L_{\zeta} \Delta \zeta
$$

In order to compare aerodynamic derivatives, with other aircraft, these are non-dimensionalised. So, the rolling moment derivatives are defined as:

$$
C_{l_{i}}=\frac{I_{x x}}{\bar{q} S b} L_{i} ; \quad i=p, \xi, r, \beta, \zeta
$$

The limits of these approximate descriptions are defined by model fidelity (or quality of flight test data). Accepting linear simplifications around a given trim point, aerodynamic derivatives are only valid within small deviations around the original trim point. To accomplish a sufficiently accurate flight simulation over a wide flight envelope, a sufficiently large database must be obtained.

\section{Identification of aerodynamic derivatives}

Results from the $\mathrm{CA}^{2} \mathrm{LM}$ framework simulations must be converted to aerodynamic derivative form to quantify the effect of wingtip morphing on the lateral dynamics. This allows for a more general comparison with literature and compatibility with other flight simulators. In order to do so, a parameter identification (PI) method is used to process the outputs of each simulation and derive the aerodynamic derivatives of interest. Through a simple ordinary least squares (OLS) approach, derivatives are obtained using the SIDPAC library. ${ }^{15}$ A more detailed description of the process is given in Dussart et al. ${ }^{3}$ In aerodynamic derivative form, the preliminary investigation on wingtip morphing effect highlighted shifts in $C_{l_{p}}$, roll damping or roll derivative due to roll rate, $C_{l_{\xi}}$, roll derivative for aileron effectiveness and $C_{l_{\beta}}$, roll derivative due to sideslip. Results have shown that aeroelastic affects the amplitude of changes to aerodynamic derivatives due to the morphing wingtip. In fact, greater changes are introduced when the aircraft is assumed to be rigid compared to the flexible configuration. Predicted changes in aerodynamic coefficients are relatively small for $C_{l_{p}}$ and $C_{l_{\xi}}$ whilst more significant changes in $C_{l_{\beta}}$ are found at lower dynamic pressures. These results point towards the need for pilot-in-the-loop simulations. 


\section{Real time flight simulation framework}

As an engineering flight simulator, the EFS500 was designed to help study aircraft stability \& control behaviour through real time flight simulations. This makes the simulator an ideal tool for rapid development of aircraft concepts, avionics and flight control systems. ${ }^{16}$ The underlying code and modelling theories used in the simulator software are extensively described in the dedicated literature. ${ }^{17}$ Access to aicraft models and characteristics (including aerodynamic derivatives) is relatively easy, making it a suitable tool for this flight dynamic investigation.

The simulator consists of a cockpit environment with a generic cockpit layout that consists of a central control stick, rudder pedals, a side-stick, a throttle lever and additional controls for various aircraft parameters. A set of networked computers run the flight dynamic models, displays, avionics, an instructor station and manage the audio cueing system. An image generation system relying on three projectors and a spherical screen provides the pilot with out-the-window scene. The reader is referred to the dedicated literature ${ }^{18}$ for more details whilst a general illustration is given in Fig.6.

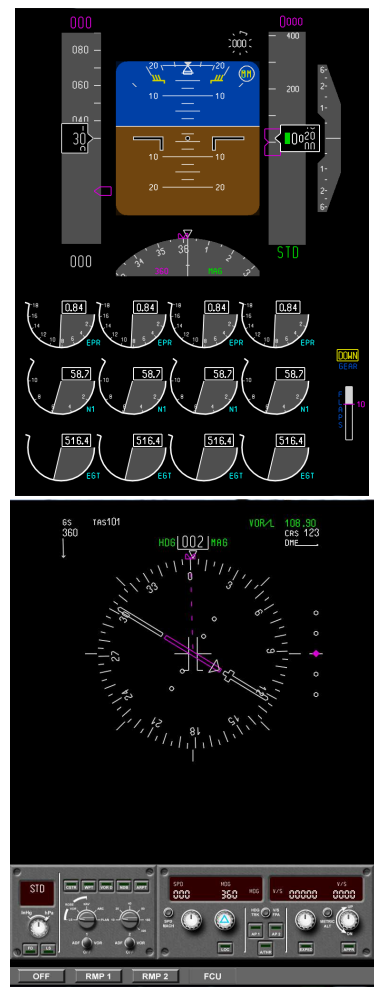

(a) Primary displays

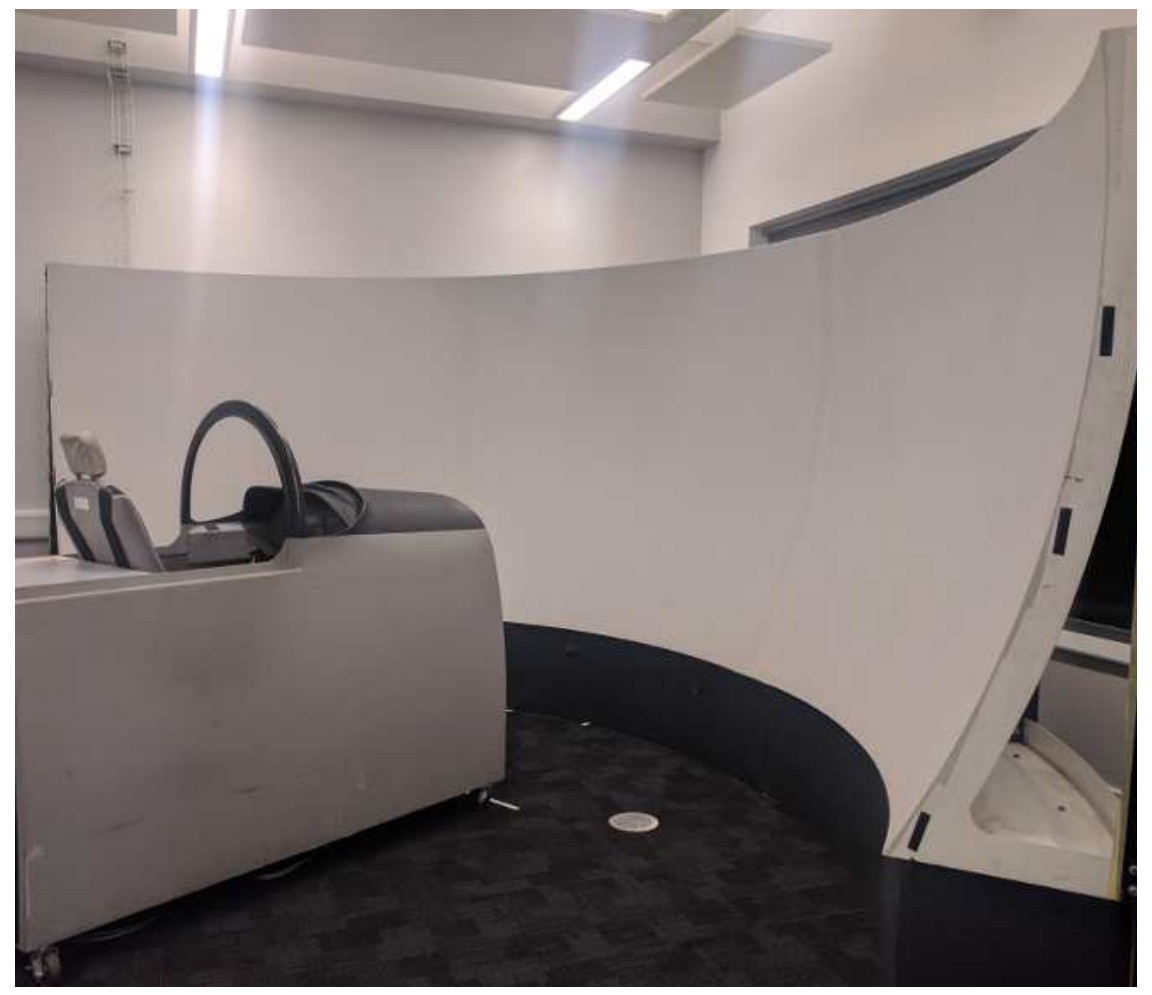

(b) Overview of the simulator hardware setup

Figure 6: Example of EFS500 cockpit displays and picture of the general setup.

An aircraft similar to the AX-1 aircraft (as presented in Section II) is implemented in the EFS500 simulator. Assuming that flap deflection is zero and the yaw damper is enabled, the relevant stability derivatives values are given in Table 1 and are comparable to those found in past work. ${ }^{3}$ These values have to be modified to account for the folding wingtip at any flight condition. For convenience, modifications are made using an increment $(\Delta)$ on top of the original aircraft values. Hence, the variation in roll damping can be written as :

$$
C_{l_{p}}=\bar{C}_{l_{p}}+\Delta C_{l_{p}}
$$

where $C_{l_{p}}$ is the total coefficient, $\bar{C}_{l_{p}}$ is the original model coefficient and $\Delta C_{l_{p}}$ is the modification applied to the model. Similar equations are used to model variations to $C_{l_{\xi}}$ and $C_{l_{\beta}}$. 


\begin{tabular}{llll}
\hline \hline Model & $C_{l_{p}}\left(\mathrm{rad}^{-1}\right)$ & $C_{l_{\xi}}\left(\mathrm{rad}^{-1}\right)$ & $C_{l_{\beta}}\left(\mathrm{rad}^{-1}\right)$ \\
\hline \hline EFS-500 & -0.4 & 0.0403 & -0.2 \\
$\mathrm{CA}^{2} \mathrm{LM}+$ SID (rigid) & -0.45 & 0.07 & -0.2 \\
$\mathrm{CA}^{2} \mathrm{LM}+$ SID (flexible) & -0.3 & 0.033 & -0.28 \\
\hline \hline
\end{tabular}

Table 1: Aerodynamic derivative values for the considered aircraft model at comparable sea level conditions

\section{Simulation task definition and post-processing methods}

\section{A. Simulation metrics and post-processed data}

Before defining manoeuvres and pilot tasks, it is important to define experimental parameters. Two types of measurements can be defined in flight simulation and testing : (1) qualitative pilot opinion and feedback and, (2) quantitative flight measurements.

To efficiently use pilot feedback regarding aircraft handling qualities, the Cooper-Harper rating method ${ }^{19}$ (CHR) was developed based on many years of flight testing. It requires the pilot to assess if a specific task or manoeuvre is achievable, and how much workload is required. Ratings are divided between desirable, acceptable and undesirable performances. In the scope of this research, a CHR for each task will provide a quick and methodical feedback from the pilots, their impression on the impact of wingtip morphing on flight dynamics. With a sufficient number of pilots, it is possible to obtain a viable set of opinions and therefore, useful qualitative feedback on the handling qualities, averaging possible discrepancies due to personal preferences. ${ }^{4}$

It is also possible to use post-processed flight simulation data to obtain time histories of key variables. Usually, aircraft flight dynamic variables and pilot inputs are stored and used for analysing controllability and pilot workload during simulation. The EFS500 can be configured to display and record numerous variables from the instructor's station. The flight simulator engineer can hence assess the quality of the manoeuvre before exporting the data for analysis. Overall, the quantitative results will be compared to task and certification requirements, ${ }^{5}$ to be used for assessing the pilot's control aggressiveness and workload throughout the task.

\section{B. Test campaign procedure}

Pilot-in-the-loop simulations are particular in the sense that the pilot will not only respond to a disturbance using feedback from the environment and the aircraft, but also use his/her past experience. More specifically, human operators demonstrate highly non-linear control behaviour, ${ }^{13}$ especially when faced with demanding tasks and high order dynamics. Pilots often display pulsive behaviour in an attempt to simplify the required control effort. ${ }^{20}$ The effect of training on pilot capability to reject disturbances has also been highlighted. ${ }^{13}$ A drastic effect on pilot performance during flight test will depend on the number of practice runs and test duration.

Therefore, it is important to clearly define the methodology and expectations of any tests with planning and discipline. ${ }^{7}$ It will greatly facilitate the post processing time, operational task of the simulation engineer and optimise simulation time to prevent repetition or degradation of acquired data. Task definition must include detailed descriptions and limits for a number of parameters such as objectives, manoeuvre targets and tolerances, limitations, test conditions and number of practice runs. Each manoeuvre should be kept as simple as possible whilst : (a) capturing the correct dynamics to facilitate the piloting task, (b) prevent possible drifts from optimal trajectory and (c) reduce post-processing time. For the pilot's assessment to be as unbiased as possible, the pilot will be given a number training time and trial runs for each manoeuvre. Simple tasks should be used as training, whilst continuously recording data. Post processing will provide an insight on possible changes of pilot behaviour over the simulation session.

\section{Definition of training tasks}

The following set of tasks were selected as training manoeuvres for this investigation, with the help of visiting test pilots and flight test engineers. 
1. Bank Angle Capture / Steady Turn Manoeuvre:

After trimming the aircraft, the pilot is required to reach a bank angle target, stabilise and hold. This will initialise a steady turn. Measurements of roll rate, time to target bank angle and pilot control inputs will be made for 3 different target bank angles at different flight conditions. Two approaches to the manoeuvre will be considered :

(a) Reach desired bank angle target within time limits

(b) Reach desired bank angle as quickly as possible (with full control input)

This task was conducted first as a training manoeuvre because it focuses solely on the lateral dynamics of the aircraft and gives the pilot an initial appreciation of aircraft dynamics.

2. Combined Symmetric Bank Angle Capture and Hold Manoeuvre :

As described in Fig.7, a roll motion is initiated to meet a target bank angle. Attitude is then held for 5 seconds before aiming for a symmetrical bank target. It is then restored after a 5 second hold. The pilot is encouraged to use maximum deflection when changing bank attitude. This more complete lateral motion task is inspired by AMC 25.147(d) (Lateral Control : Roll Capability ${ }^{5}$ task) and gives satisfactory insights on banking requirements for certification purposes. The shift from the highest bank attitudes must be made within 11 seconds.

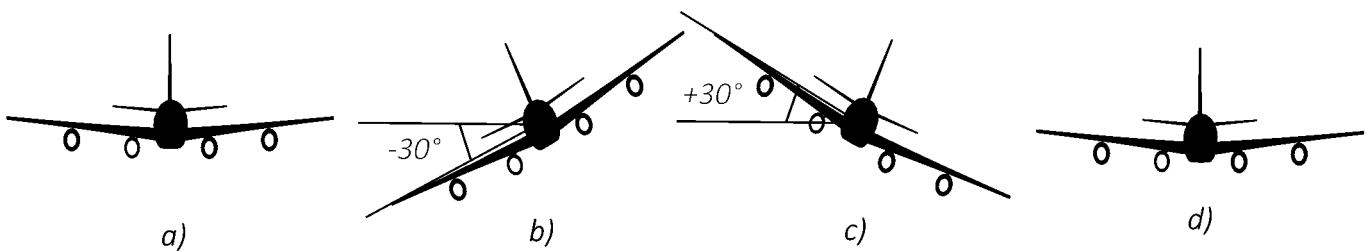

Figure 7: Illustration of the Combined Symmetric Bank Angle Capture and Hold Manoeuvre

\section{Definition of handling quality assessment tasks}

Similar to the training manoeuvres, visiting test pilots and flight test engineer's participated in the elaboration of the following set of manoeuvres:

1. Offset Landing Manoeuvre (OLM):

Illustrated in Fig. 8 and originally outlined and used by Lee, ${ }^{10}$ this scenario corresponds to an offset ILS approach for landing with a recovery manoeuvre at specific altitude. Coupling all aircraft dynamics, it is expected to be the most challenging for the pilot. It is a widely known and understood manoeuvre that test pilots requested to be included for completeness.

The OLM task requires specific capabilities from both the simulator and test pilot. The pilot must be capable of landing the aircraft based on the instruments and the ILS indicator ${ }^{\mathrm{a}}$. It also requires a landing gear model, ground interaction model, and an ILS indicator. But most importantly, this manoeuvre can realistically be made only in landing flight conditions.

2. Slalom and Alignment Tracking Manoeuvre (SLAT):

A slalom manoeuvre, requiring symmetric banking turns, was defined to avoid the need for expansive modelling required for the OLM task. Coupled with alignment tracking and positioning cues, or gates, this manoeuvre can be flown at multiple speeds and altitudes, wing configurations, with CHR ratings and simulation data analysis. This manoeuvre is illustrated in Fig.9a, and described below in more details. 


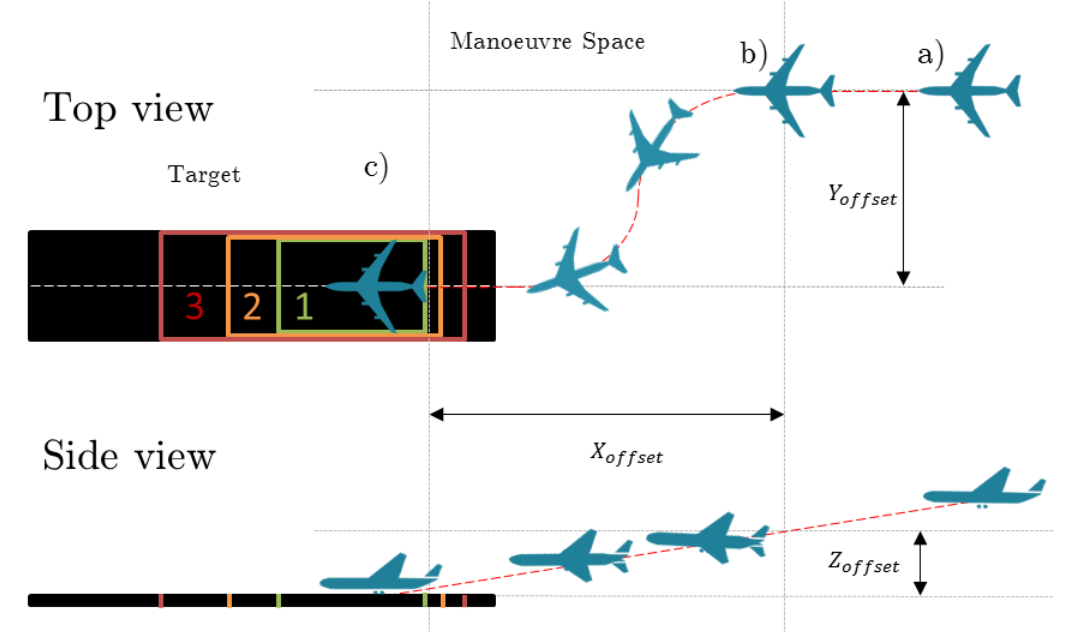

Figure 8: Illustration of the Offset Landing Manoeuvre, with desired touch down positions on the runway and initial starting point.
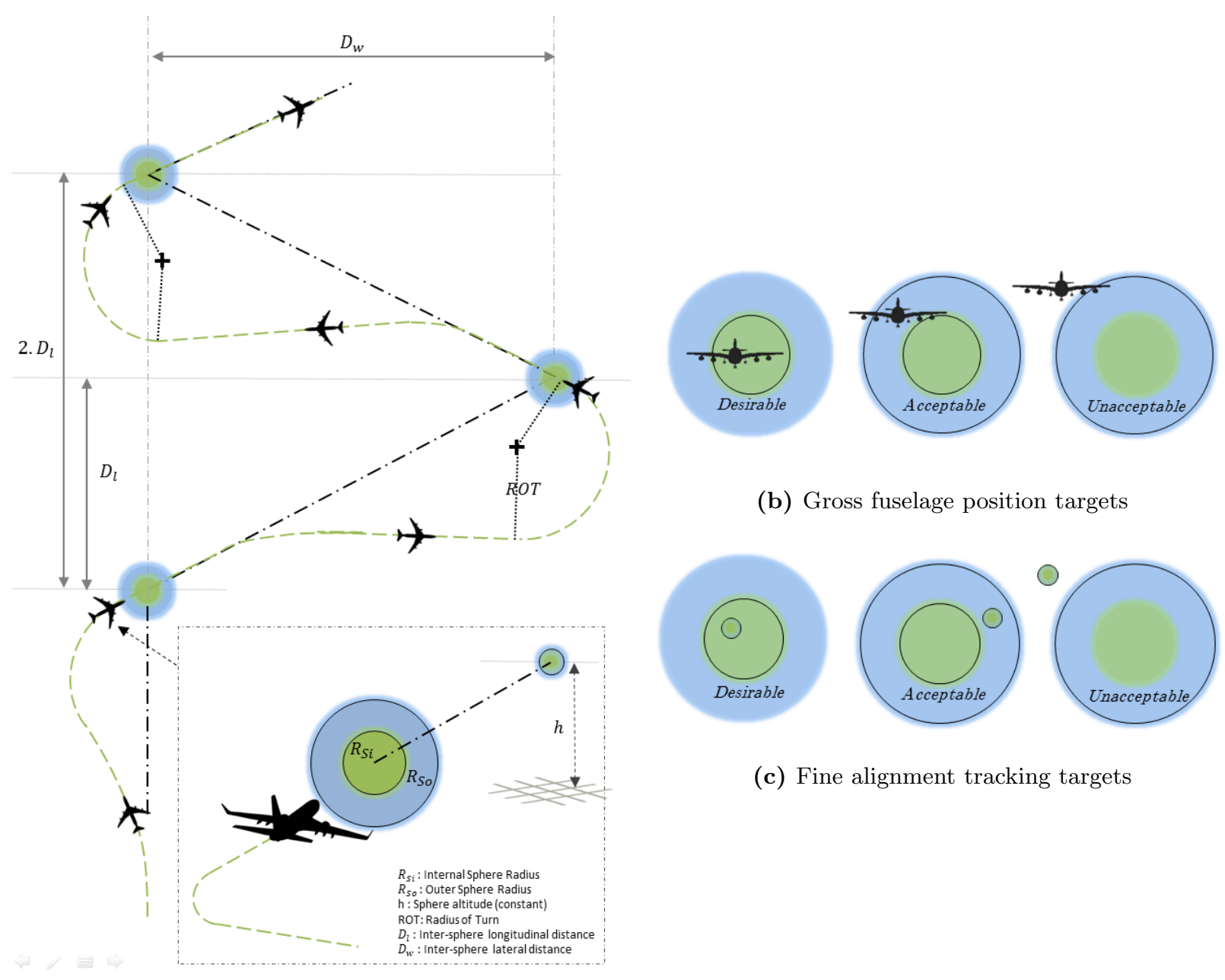

(b) Gross fuselage position targets
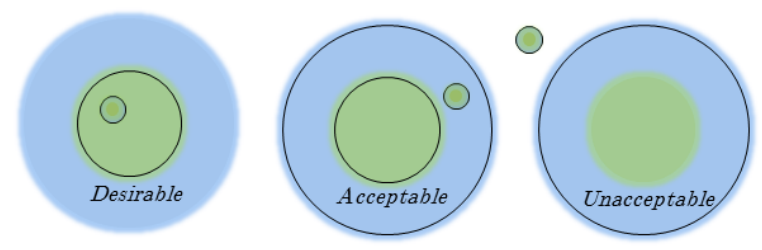

(c) Fine alignment tracking targets

(a) Manoeuvre illustration

Figure 9: Illustration of the Slalom and Alignment Tracking task and targets for CHR 
The SLAT task was designed as a multi-axis manoeuvre to assess longitudinal, lateral and directional dynamics. It was designed to evaluate specific mission capabilities: gross position and fine alignment tracking. The pilot is tasked to fly through a set of checkpoints positioned in a slalom at constant altitude. He/she must also strive to align the next gate prior to the checkpoint pass within acceptable boundaries, as displayed in Fig.9a. Visual targets are used to guide the pilot throughout the manoeuvre. Multiple groups of two concentric transparent spheres, of different radii and color are used to model the gates. Size and position of the spheres are based on the aircraft characteristics, altitude and airspeed to ensure that the manoeuvre is feasible in realistic flight conditions (limited banking angle load factors). The turn radius for a given maximum bank angle and realistic trajectory are calculated using a simplified state-space model, coupling longitudinal, lateral and directional dynamics based on aerodynamic derivatives of the baseline aircraft. These are then used to scale distances between spheres, $D_{w}$ and $D_{l}$ as shown in Fig.9a. To capture both mission capabilities, two parameters are defined to quantify the suitability of the manoeuvre. The first focuses on the aircraft position at gate checkpoints, and helps in the assessment of pilot effort to reach a target position. The other focuses on the aircraft attitude and flight path when reaching the gate, and requires a more fine tuning and control of the aircraft.

To help the pilot and operators assess if the simulation is suitable, three different boundaries are defined for both criterias. These boundaries are clearly visible during the simulation, and are based on the checkpoint sphere radius (scaled so that gates are sufficiently visible). The task is both achievable and sufficiently challenging for all test conditions. Internal Sphere Radius $\left(R_{S i}\right)$ was set to $50 \mathrm{~m}$, whilst Outer Sphere Radius $\left(R_{S o}\right)$ was set to $100 \mathrm{~m}$. As the first criteria focuses on the aircraft position when flying through the gate, centre of gravity of the vehicle is evaluated, as shown in Fig.9b where the desirable, acceptable and unacceptable boundaries are illustrated. The second criteria focuses on the capability of the pilot to reach and maintain a specific attitude and position, so as to align the vehicle with given targets. Hence, the pilot must use both current and next gates as shown in Fig.9c to judge if the aircraft alignment was desirable, acceptable or unacceptable when crossing the gate. The pilot is then tasked with completing a CHR for each criteria, directly after the manoeuvre. Flight data recording of aircraft position and attitude, as well as pilot provides confirmation of gross position and alignment ratings.

\section{Results and Discussion}

A number of preliminary flight simulations were conducted with the EFS500 simulator. The aircraft model was updated during simulation through the operator station for a range of $\Delta C_{l_{p}}$ and $\Delta C_{l_{\xi}}$ values. Acceptable limits to $\Delta C_{l_{p}}$ and $\Delta C_{l_{\xi}}$ were found to be approximately \pm 0.1 and \pm 0.02 respectively when tested independently in the challenging offset landing manoeuvre. The boundary values defined from pilotin-the-loop tests include the maximum shifts found in past work, ${ }^{3}$ as shown in Table 2. It is important to note that shifts in aerodynamic derivatives are instantaneous, and no transient regime is modelled in the flight simulator.

\begin{tabular}{lccc}
\hline \hline Source & $\Delta C_{l_{p}}\left(\mathrm{rad}^{-1}\right)$ & $\Delta C_{l_{\xi}}\left(\mathrm{rad}^{-1}\right)$ & $\Delta C_{l_{\beta}}\left(\mathrm{rad}^{-1}\right)$ \\
\hline \hline Preliminary testing (EFS-500) & $-0.10,+0.10$ & $-0.020,+0.020$ & $-0.10,+0.10$ \\
$\mathrm{CA}^{2} \mathrm{LM}+$ SID (rigid) & $-0.08,+0.00$ & $-0.004,+0.000$ & $-0.05,+0.00$ \\
$\mathrm{CA}^{2} \mathrm{LM}+$ SID (flexible) & $-0.01,+0.02$ & $-0.004,+0.000$ & $-0.01,+0.00$ \\
\hline \hline
\end{tabular}

Table 2: Maximal range of aerodynamic derivative shifts considered for testing

Particular care was taken in the verification and validation of the SLAT manoeuvre for handling quality assessment. The manoeuvre was flown at multiple flight conditions and gate spacing. Time histories of aircraft states, pilot inputs and aircraft trajectories are valuable post processing results for flight dynamic analysis and handling qualities assessment. Both can be used to quickly assess the suitability of the manoeuvre, as shown in Fig.10 where an example of an overall undesirable SLAT manoeuvre is given. In this case, it can be seen that the alignment criteria was not respected in both gates 2 and 3, despite a desirable

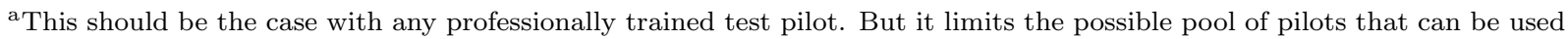
for the simulations. As only flight test pilots can give reliable CHR ratings, other less experimented pilots can be used for a quantitative analysis.
} 


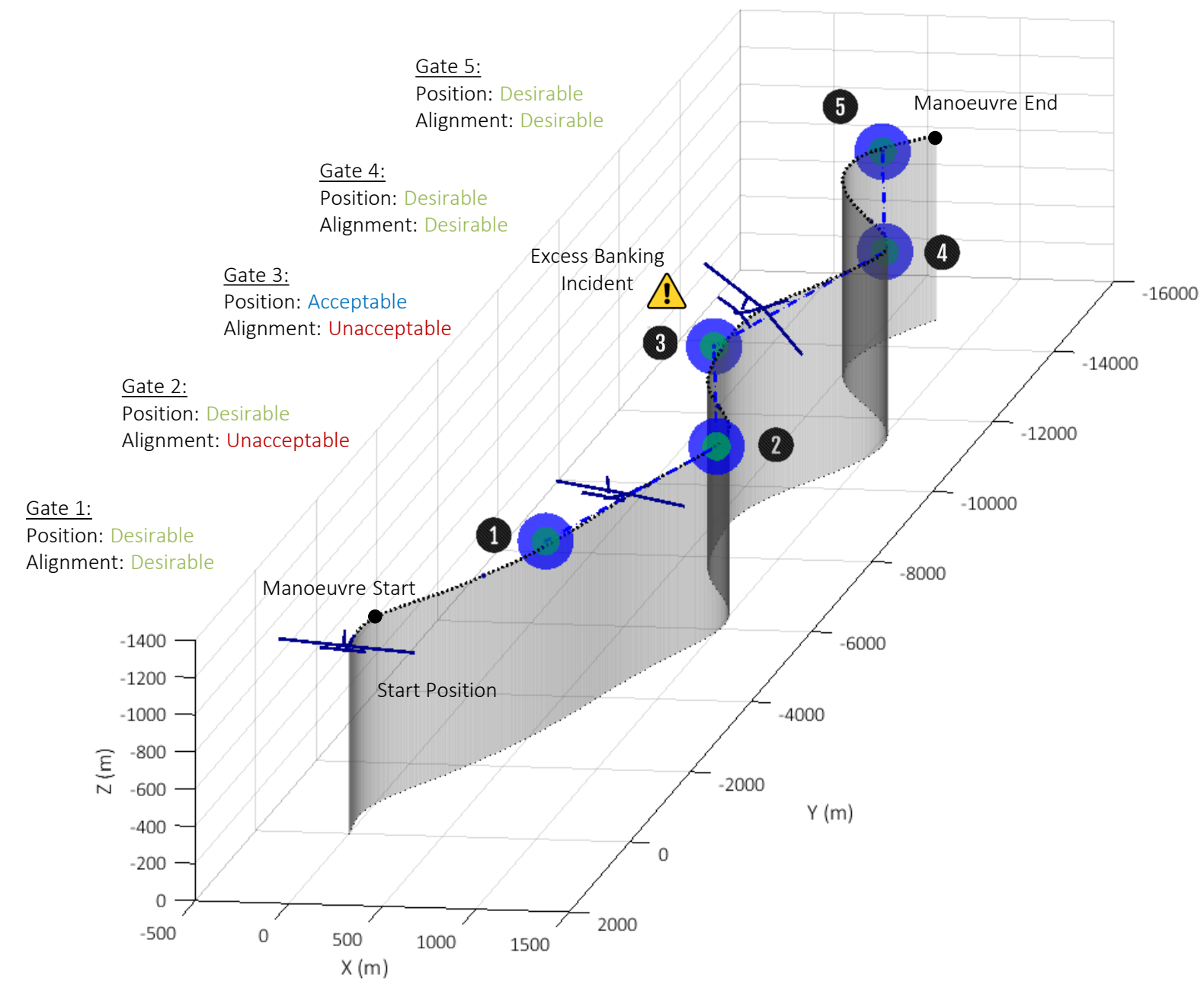

Figure 10: Annotated trajectory illustration of a SLAT manoeuvre example

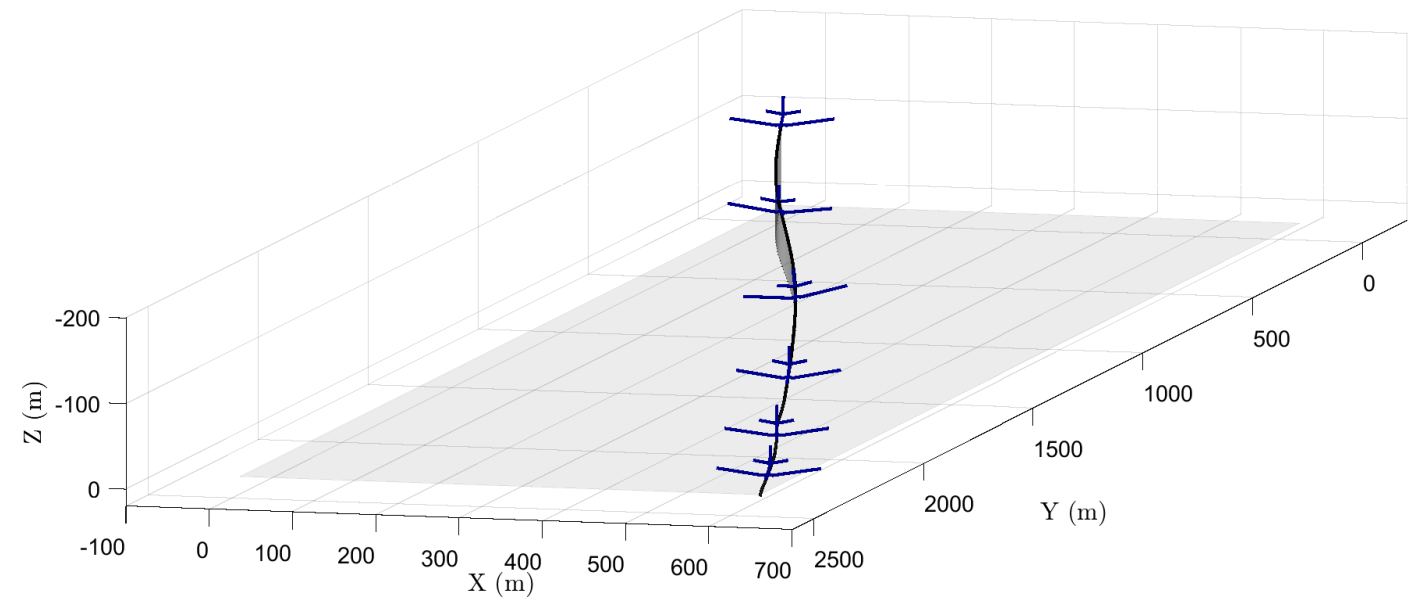

Figure 11: Trajectory illustration of an OLM manoeuvre example 
centre of gravity position during the manoeuvre (first criteria). It can also be seen that during the flight segment between gates 3 and 4, excessive banking (over the manoeuvre $45^{\circ}$ limit) was reached, as shown in the time histories given in Fig.12a. In this particular test, the effect of airspeed on manoeuvre feasibility is also clearly highlighted, since the pilot failed to maintain steady airspeed. As airspeed increases, higher banking angles and roll rates are required to reach target position and alignment, leading to an excessively aggressive manoeuvre in the end. Therefore, particular care must be taken to force constant airspeed on the aircraft when flying this manoeuvre.

The OLM manoeuvre was also verified and validated as a suitable manoeuvre for the handling quality assessment of the morphing aircraft. The pilot was tasked to complete the manoeuvre at design landing conditions (flaps and undercarriage deployed, landing speed) with various aerodynamic derivative shifts before completing a CHR scale. The aircraft touchdown position, clearly visible in the time histories provided in Fig.12b is used to assess the validity of the manoeuvre and respect of the landing position criteria. In the baseline example illustrated in Fig.11, the landing position criteria was met, with an acceptable workload from the pilot. Subsequent CHR rating also pointed to satisfactory performances. The impact of morphing on aerodynamic derivatives led to different responses, making the task more challenging as the aileron efficiency was reduced.

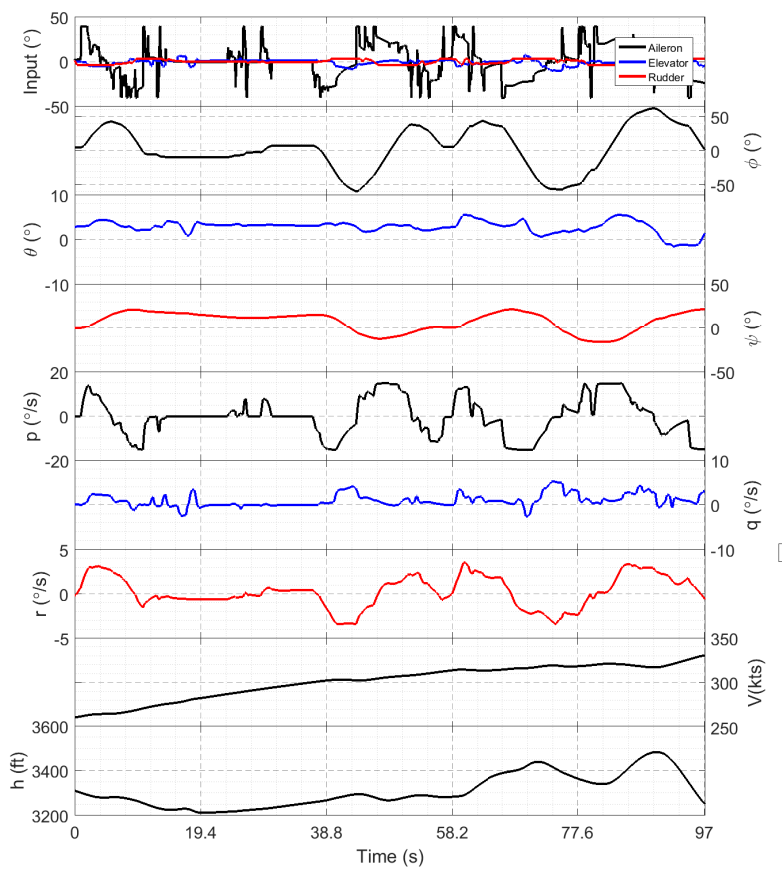

(a) SLAT manoeuvre from Fig.10

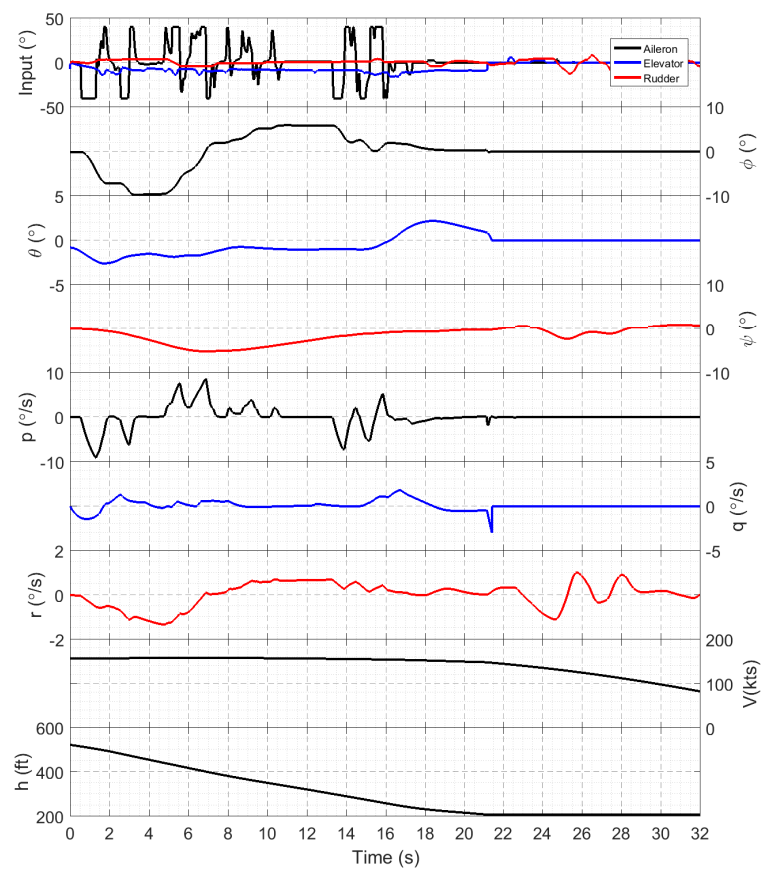

(b) OLM manoeuvre from Fig.11

Figure 12: Time history examples

\section{Conclusions and Further Work}

In past work, the effect of morhing wingtips on aircraft lateral dynamics was identified using $\mathrm{CA}^{2} \mathrm{LM}$, a 6 DoF aeroservoelastic framework and a system identification process. By matching $\mathrm{CA}^{2} \mathrm{LM}$ non-linear simulation outputs and the linear identification model, the effect of morphing on key lateral aerodynamic derivatives was identified. ${ }^{3}$ These results highlighted the need for pilot-in-the-loop simulations to investigate possible changes to the morphing aircraft handling qualities.

The simulators and methodologies used to carry out this investigation were presented in this paper. Modifications were made to the engineering flight simulator to allow for real-time changes in aerodynamic properties. The $\mathrm{CA}^{2} \mathrm{LM}$ framework was also modified to include cockpit and computation acceleration hardware. ${ }^{18}$ The assessment of the morphed aircraft handling qualities was made using both qualitative 
and quantitative data measurements of multiple manoeuvres. This list of manoeuvre was carefully selected to initiate correct motion corresponding to relevant aerodynamic coefficients. Procedure and experimental conditions were developed with the help of experienced flight test pilots and engineers. Special care was taken to limit human factor uncertainty such as pilot aggressiveness, personal preferences and experience by clearly defining the experimental procedure of the simulation campaign. A Slalom and Alignment Tracking manoeuvre is also introduced. This manoeuvre was designed as a multi-directional task, tailored for different flight speeds and altitudes depending on aircraft characteristics and acceptable turn rates. Targets in aircraft gross position and alignment at different checkpoints were defined. Using the morphing test case, this manoeuvre proved to be an insightful method to assess the lateral-directional handling qualities of the aircraft over an extended flight envelope. The widely used Offset Landing Manoeuvre was also included, but provides results only in landing configuration.

From pilot simulations, it was found that changes to aircraft aerodynamic derivatives due to morphing led to performances within the acceptable boundaries defined by the preliminary simulations with a licensed pilot. Hence, pilot feedback and simulation measurements indicate that little to no changes are required to reach target response. This is very encouraging in the effort to de-risk the in-flight morphing mechanism, if it were to be capable to reach desired morphing characteristics. For a thorough handling qualities investigation, a larger batch of pilots, including experienced test-pilots have been invited to participate in the further development of this research. This will lead to a revised acceptable performance assessment along with possible wingtip mechanism redesign or control scheme guidelines.

\section{Acknowledgments}

This work is supported and developed in collaboration with Airbus Group, Innovate UK and the Aerospace Technology Institute (UK ATI). The authors would also like to acknowledge the support of the Indonesia Endowment Fund for Education (Lembaga Pengelola Dana Pendidikan - LPDP).

\section{References}

${ }^{1}$ A. Castrichini, V. H. Siddaramaiah, D. E. Calderon, J. E. Cooper, T. Wilson, and Y Lemmens. Nonlinear Folding Wing-Tips for Gust Loads Alleviation. Journal of Aircraft, 53(5):1391-1399, 2015.

${ }^{2}$ David Hayes, Mudassir Lone, James Whidborne, and Etienne Coetzee. Evaluating the Rationale for Folding Wing Tips Comparing the Exergy and Breguet Approaches. In 55th AIAA Aerospace Sciences Meeting, 2017.

${ }^{3}$ Gaetan Dussart, Sezsy Yusuf, and Mudassir Lone. Effect of wingtip morphing on the roll mode of a flexible aircraft. In AIAA Agile Wing Interactions, AIAA SciTech Forum, Orlando, Florida, 8-12 January 2018.

${ }^{4}$ J. C. Gibson. The definition, understanding and design of aircraft handling qualities. Technical report, Delft University of Technology, Delft, 1995.

${ }^{5}$ Certification Specifications and Acceptable Means of Compliance for Large Aeroplanes CS25, 2012

${ }^{6}$ Airworthiness Standards: Transport Category Planes, 2013.

${ }^{7}$ D.T. Ward and T.W. Strganac. Introduction to flight test engineering. Kendall/Hunt Publishing Company, 1996.

${ }^{8}$ Marty K. Bradley and Christopher K. Droney. Subsonic Ultra Green Aircraft Research: Phase II. Technical report, 2012.

${ }^{9}$ The Boeing Company - Boeing 777X. http://www.boeing.com/commercial/777x/gallery, 2017. Accessed 1/12/2017.

${ }^{10}$ B.P. Lee. Recent experience in flight testing for pilot induced oscillations (PIO) on transport aircraft. The Aeronautical Journal (1968), 2000.

${ }^{11}$ S.P. Andrews. Modelling and simulation of flexible aircraft: handling qualities with active load control. PhD thesis, Cranfield University, School of Engineering, 2011.

${ }^{12}$ Vilius Portapas, Alastair Cooke, and Mudassir Lone. Modelling framework for flight dynamics of flexible aircraft. Aviation, 20(4):173-182, 2016.

${ }^{13}$ Mudassir Lone. Pilot modelling for airframe loads analysis. PhD thesis, Cranfield University, 2013.

${ }^{14} \mathrm{M}$. V. Cook. Flight dynamics principles: a linear systems approach to aircraft stability and control. Elsevier aerospace engineering series. Butterworth-Heinemann, Amsterdam ; Boston, 3rd ed edition, 2013.

${ }^{15}$ E. Morelli. System identification programs for aircraft (SIDPAC). AIAA Atmospheric Flight Mechanics Conference and, 2002.

${ }^{16}$ David J. Allerton. The design of a real-time engineering flight simulator for the rapid prototyping of avionics systems and flight control systems. Transactions of the Institute of Measurement, 1999.

${ }^{17}$ David J. Allerton. Principles of flight simulation. Wiley, 2009.

${ }^{18}$ Guillermo Lopez, Vilius Portapas, Gaetan Dussart, Mudassir Lone, and Etienne Coetzee. Pilot-in-the-loop flight simulation of flexible aircraft in matlab/simulink: Its implementation and coding peculiarities. In AIAA Model-Based Development and X-in-the-Loop Simulation Conference, AIAA SciTech Forum, Orlando, Florida, 8-12 January 2018.

${ }^{19}$ G.E. Cooper and R.P. Jr Harper. The use of pilot rating in the evaluation of aircraft handling qualities. Technical report, NASA TN D-5153, Washington D.C., April 1969. 
${ }^{20}$ Ronald A. Hess. A Rationale for Human Operator Pulsive Control Behavior. Journal of Guidance, Control, and Dynamics, 2(3):221-227, may 1979. 
2018-01-13

\title{
Method to assess lateral handling qualities of aircraft with wingtip morphing
}

\author{
Dussart, Gaétan X.
}

Gaétan X. Dussart, Sezsy Y. Yusuf, Vilius Portapas, et. al., Method to assess lateral handling qualities of aircraft with wingtip morphing. 2018 AIAA Atmospheric Flight Mechanics

Conference, 8-12 January 2018, Kissimmee, FL, USA. Paper number AIAA 2018-1015

http://dx.doi.org/10.2514/6.2018-1015

Downloaded from Cranfield Library Services E-Repository 